\title{
Vlasov Launcher Diagrammatic Design Using the RT Method
}

\author{
Andrzej Francik, Grzegorz Jaworski, Maciej Nowak, and Kacper Nowak \\ Faculty of Electronics, Wrocław University of Technology, Poland
}

\begin{abstract}
In this paper, a simple and fast method relied upon for designing a Vlasov launcher with a helical cut is proposed. The method is based on graphic interpretation of analytical relationships that link wave parameters (EM field mode) to the launcher's geometrical dimensions. Using the ray tracing method, a simplified graphic analysis may be carried out. The results obtained are not significantly different from those of rigorous full-wave analyzes. The family of normalized curves that is created in the process greatly facilitates the stage of optimizing the geometrical parameters of the Vlasov launcher.
\end{abstract}

Keywords—gyrotron output, ray tracing, Vlasov launcher.

\section{Introduction}

Over the past decade, interest in the gyrotron technology has increased significantly due to its potential applications in many fields, such as plasma heating, material processing, radar and communications systems, and medical research. One of the most promising fields is that of fusion energybased power generation. A mode converter is a key component enabling efficient RF propagation in a high power gyrotron. In recent years, several designs of a quasi-optical mode converter have been presented [1]-[4]. The quasioptical mode converter with a Vlasov launcher is still incorporated in modern gyrotron designs.

Bian et al. presented a broadband quasi-optical mode converter that could function at three segment-continuous frequencies in three different waveguide modes [5]. Gao et al. described the design and fabrication of a frequency-agile gyrotron used for frequency-chirped MAS DNP [6]. Alaria et al. described the design of a helical cut, smooth-wall Vlasov launcher for converting the $\mathrm{TE}_{22,6}$ mode to a Gaussian mode, used in a $120 \mathrm{GHz}, 1 \mathrm{MW}$ gyrotron [7]. Zhang et al. described a study on a gyrotron with a quasi-optical mode converter for terahertz imaging [8].

The Vlasov launcher still appears to be attractive for designing conventional high-power gyrotrons operating in the high terahertz range. At this frequency range, the complicated structure of a Denisov launcher makes precise design and manufacturing processes increasingly difficult. A properly optimized internal converter based on a Vlasov launcher is compatible with the gyrotron's electro-optical system and may generate a clear Gaussian beam with an efficiency of more than $80 \%$ in an extraordinarily broadband range [1]. A further correction with the use of mirrors allows to obtain the efficiency of up to $90 \%$ [9].

For the design of a Vlasov launcher, approaches based on the geometric optics theory and specialized software, such as electric field integral equation code (SURF3D LOT/SURF-3D) are frequently used [10]. Full-wave electromagnetic simulation software, such as CST, is used as well [7], [8]. This article presents an analysis of propagation of electromagnetic waves in the gyrotron's internal components. The results of such an analysis serve as a basis for developing an effective launcher design procedure. The theoretical foundations of the ray tracing (RT) method are relied upon in the analysis in a manner that is presented exhaustively in [11]. The RT method is very good for designing key elements of a gyrotron, such as the waveguide (forming the input section of the launcher), the launcher, and the mirror system. It relies on much simpler mathematical formulas to describe the propagation of electromagnetic waves - especially when compared with methods based on Maxwell's field equations. The proposed design procedure was also verified, with good results, by comparing it with calculations based on data published in the literature of the subject.

The analysis of propagation of electromagnetic waves in the launcher, being the gyrotron's internal component, is presented and serves as a basis for developing an effective launcher design procedure. A Vlasov antenna [12], designed using the oversized circular waveguide technique, is commonly used as a launcher in microwave gyrotron systems.

\section{Design Procedure}

The procedure uses a graphic representation of the known analytical relationships describing RT power transmission in a launcher system and allows the designer to make optimal decisions during the design process. 


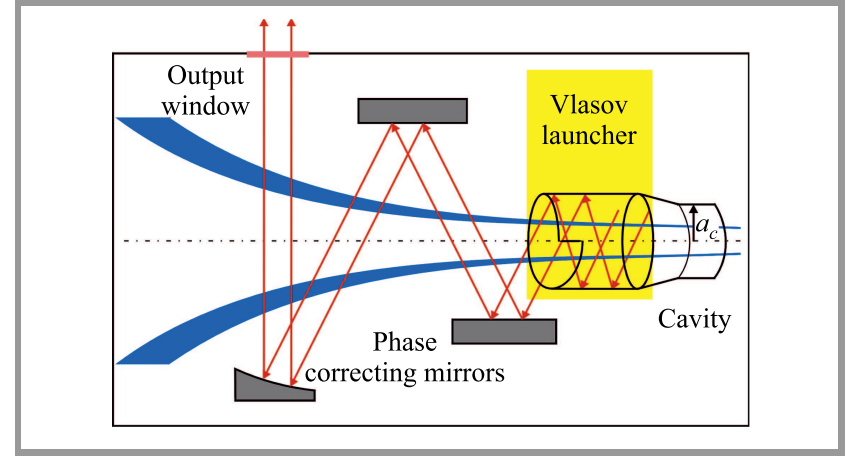

Fig. 1. Structure of the gyrotron's microwave output, with the Vlasov launcher highlighted in yellow. Blue arcs determine the spent electron trajectory, $a_{c}-$ cavity radius.

An example of a typical internal structure of a gyrotron microwave system is shown in Fig. 1. The location of the launcher within the structure is highlighted in yellow. It is usually the gyrotron cavity that is the first designed part of an internal gyrotron microwave system. It is characterized by a resonant frequency that is equal to the desired gyro frequency in which the electron beam interacts with the cavity's resonant microwave field. This interaction results in the transfer of electron beam energy into the microwave field, with its energy reaching significant power levels (measured in megawatts). In the process of cavity design, its geometrical structure is selected, as is the microwave field mode and the dimensions that are calculated taking into account the power levels generated. After designing the cavity, the microwave field mode and the geometrical parameters of the cavity may be determined. These quantities usually serve as input data required to design the next element of the structure, namely the launcher. The field types, determined in such a way, are usually highorder $T E_{m n}$ modes with $m$ and $n$ indexes whose values are much larger than 1 . This, and the ability to work at high mi-

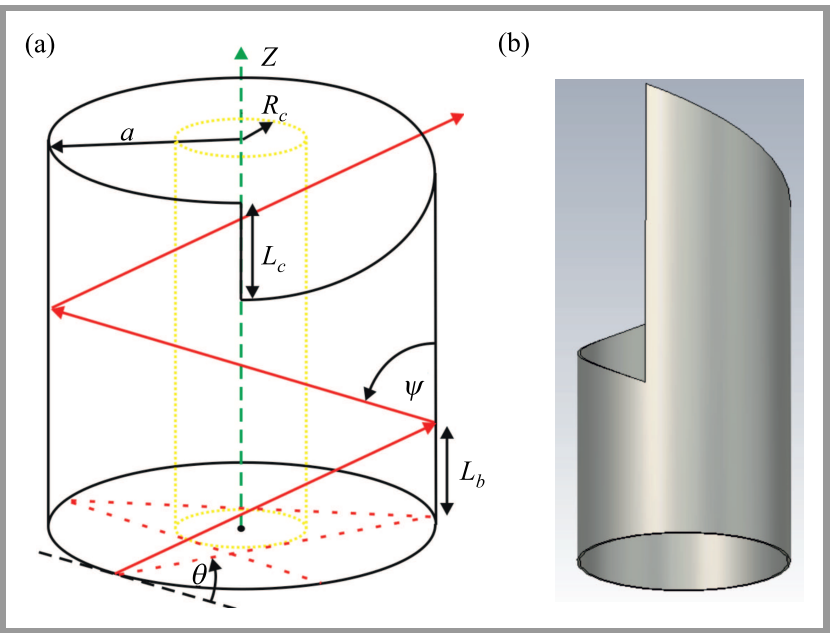

Fig. 2. Circular waveguide launcher having an $L_{c}$-length helical cut: a) ray presentation of the wave in the launcher, b) structural sketch. crowave power levels are the reasons behind using oversized microwave systems in situations in which the conditions allow to rely on the RT method. The launcher appearance and the ray presentation of the wave in the launcher are shown in Fig. 2.

In Fig. 2, most of the geometrical parameters of ray transmission (RT) are marked:

- $a$ - waveguide radius,

- $\psi-$ Brillouin angle,

- $L_{c}$ - helical cut length (the smallest possible length of the launcher $L$ ),

- $L_{b}$ - axial displacement (towards the waveguide axis) of two consecutive reflection points of the ray from the surface of the waveguide,

- $R_{c}$ - caustic radius,

- $\theta$ - azimuth angle (angle $2 \theta$ is a central angle determined on the $\mathrm{S}$ plane, perpendicular to the waveguide axis and containing one ray reflection point, defining the length of arc $2 \theta a$, where the chord is the projection of the ray on the $\mathrm{S}$ plane. The azimuth angle is clearly visible in Fig. 3, showing ray propagation of the wave beam in a circular waveguide.

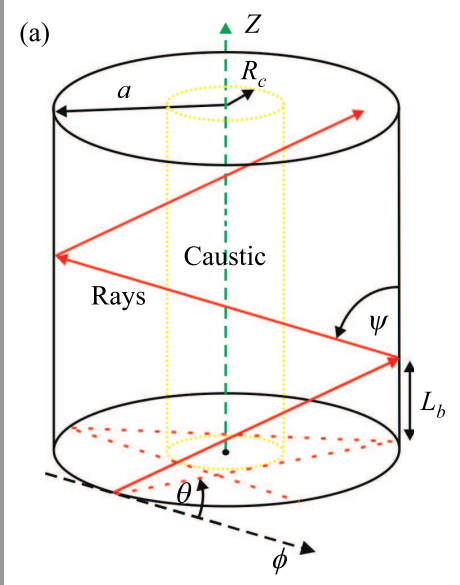

(b)

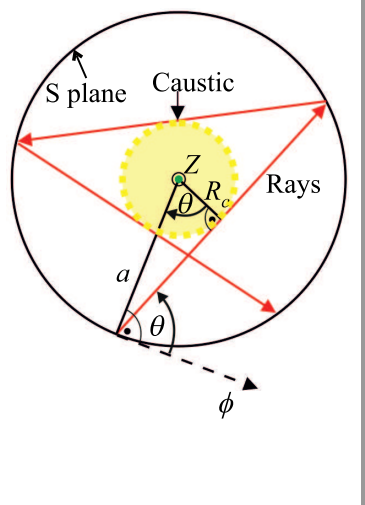

Fig. 3. Wave beam in a circular waveguide illustrated with the use of geometrical optics principle: a) side view, b) top view.

Another reflection point from the surface of the waveguide is located on the helix of the inclination angle $\tau$, created on the waveguide's surface. The inclination angle $\tau$, being another geometrical parameter of RT transmission, is visible on the unfolded surface of the launcher's waveguide, on the plane shown in Fig. 4. The straight lines, with their slopes measured in relation to the $z$ axis, equal $\tau$ and represent the helix in the plane figure, while B0-B4 parallelograms shown are the Brillouin zones. Figure 4 offers an explanation to the strategy of selecting the cutting edges. The simplest helical cut of a circular waveguide is a straight section BD. If such a cut was to be made, the entire field 
radiated from the Brillouin zone B1 would have encountered an obstacle. This would be a part of the waveguide wall designated as the Brillouin zone B2. This means that the field would obviously not be radiated into the free space. Therefore, this obstacle is removed by making a cut along the border between zones B3 and B4 and along the entire section of $\mathrm{C}^{\prime}-\mathrm{C}$.

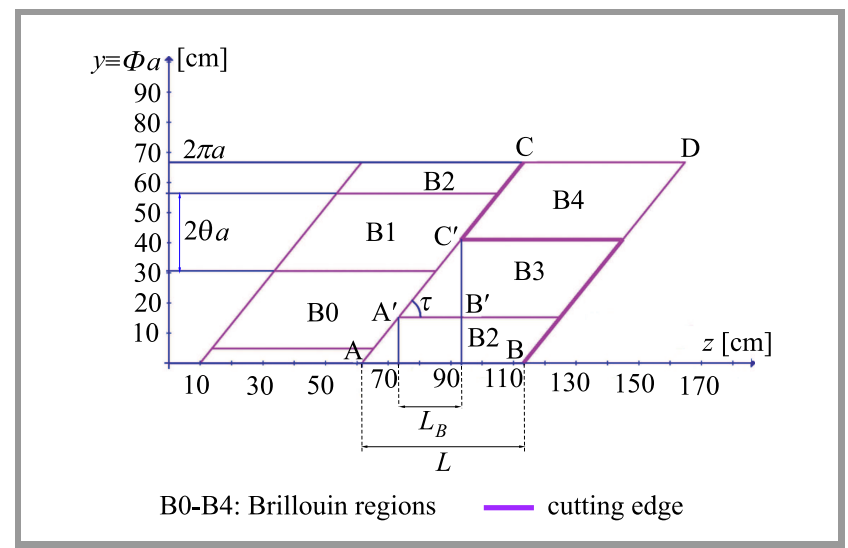

Fig. 4. The surface of a cylindrical waveguide launcher unfolded on a plane. The cutting edges of the launcher are represented using bold lines.

The RT representation shown above is satisfactorily accurate, ensuring the results of the analysis are not significantly different from the results of a rigorous full-wave analysis, when the ratio between the waveguide diameter $(D=2 a)$ and the wavelength in free space $\lambda_{0}$ is greater than 10 [13], [14]. The field radiated from the Brillouin zone, marked in Fig. 4 as B3, may be considered as a set of rays running parallel, in the axial direction, and diverging spherically in the transverse direction. These rays, propagating - for instance - towards the focal toroidal mirror, are shown in Fig. 5 on the so-called Vlasov converter used in gyrotrons to convert launcher radiation into a Gaussian beam [15].

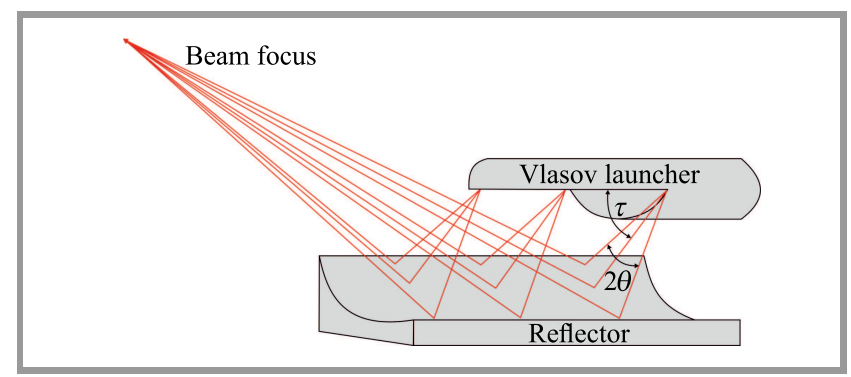

Fig. 5. Geometry of the Vlasov converter consisting of a circular waveguide launcher with helical cut and a double-curved reflector (toroid). $\tau$ is the helix inclination angle and $2 \theta$ is the radiation expansion angle transverse to the waveguide axis.

Angle $2 \theta$ marked in Fig. 5 is $v_{\text {exp }}$ and is a radiation expansion angle that is transverse to the waveguide axis. Expansion angle $v_{\text {exp }}$ is another important geometric parameter in RT transmission analysis. Mathematical dependencies describing the parameters introduced above have been formulated, inter alia, in [11]. They are based on the assumption that transmission in the launcher with the microwave structure considered here is expressed in the cylindrical coordinate system shown in Fig. 6.

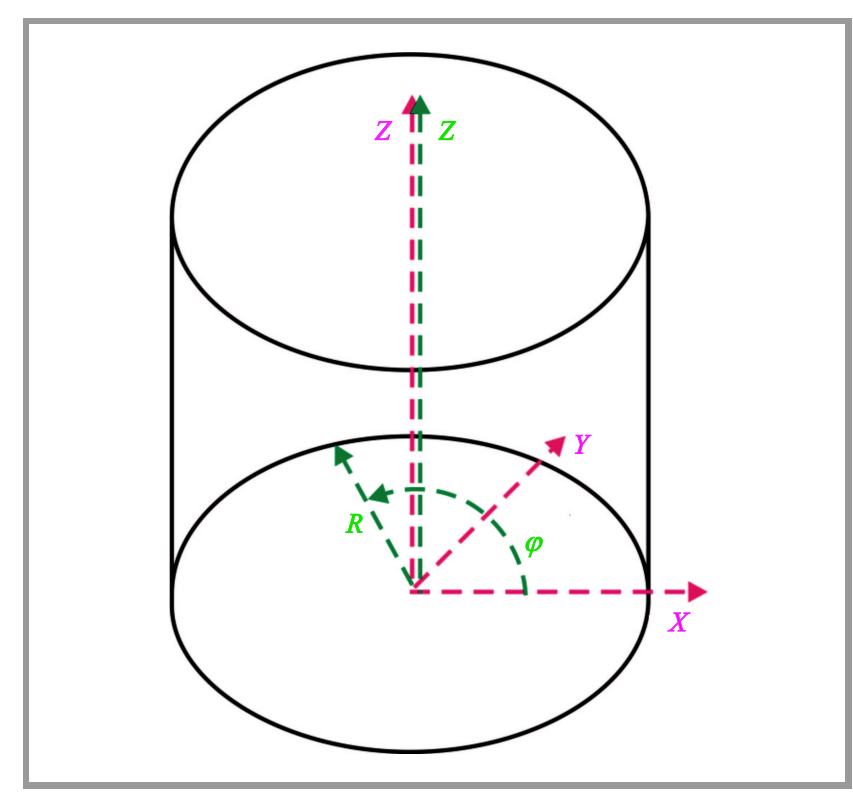

Fig. 6. The cylindrical coordinate system associated with the Vlasov launcher in such a way that cylinder axis is the axis of circular waveguide of launcher.

For the launcher and the $T E_{m n}$ mode under consideration, the $u(r, \phi, z)$ field propagating in a cylindrical waveguide, which is the input section of the launcher, may be expressed, using the cylindrical coordinate system, in the following manner:

$$
u(r, \phi, z)=A_{0} J_{m}\left(k_{r} r\right) \mathrm{e}^{ \pm \mathrm{j} m \phi} \mathrm{e}^{ \pm \mathrm{j} k_{z} z}
$$

which:

$$
\begin{array}{r}
k_{r}=\frac{\chi_{m n}}{a}, \\
J^{\prime}\left(\chi_{m n}{ }^{\prime}\right)=0, \\
k_{z}=\sqrt{{k_{0}}^{2}-k_{r}^{2}},
\end{array}
$$

where:

- $A_{0}$ - constant field amplitude,

- $z$ and $r$ - longitudinal and radial coordinate,

- $\phi$ - azimuth angle,

- $J_{m}$ and $J_{m}^{\prime} m$-th order Bessel function and its derivative,

- $\chi_{m n}$ and $\chi_{m n}{ }^{\prime} n$-th zero of $m$-th order Bessel function and its derivative,

- $k_{r}$ and $k_{z}$ - radial and longitudinal wave number,

- $k_{0}$ - wave number in free space. 
The geometrical parameters of the RT method for the considered launcher are described in the following analytical expressions:

$$
\cos \psi=\frac{\vec{N} \vec{e}_{z}}{|\vec{N}|\left|\vec{e}_{z}\right|}=\frac{k_{z}}{\sqrt{k_{r}^{2}+k_{z}^{2}}}=\frac{k_{z}}{k_{0}}, \sin \psi=\frac{k_{r}}{k_{0}},
$$

where (for TE field):

$$
\begin{gathered}
k_{r}=\frac{\chi_{m n}{ }^{\prime}}{a}, \\
k_{0}=\frac{2 \pi}{\lambda_{0}}, \\
\cos \theta=\frac{\vec{N} \vec{e}_{z}}{|\vec{N}|\left|\vec{e}_{z}\right|}=\frac{m}{\chi_{m n}}, \\
R_{c}=a \cos \theta \\
L_{B}=2 a \sin \theta \cot \psi \\
\tau=\arctan \frac{\theta \tan \psi}{\sin \theta}, \\
L=2 \pi a \cot \tau, \\
v_{\exp }=2 \theta
\end{gathered}
$$

Equations (6)-(13) allow one to design a launcher for the input data obtained from the gyrotron cavity design and from other gyrotron system requirements, such as:

- the volume of the vacuum space inside the gyrotron, that the launcher must fit in,

- trajectory of the spent electrons' motion with no launcher elements present,

- the so-called modal purity that needs to be sufficiently large, as it determines the share of Gaussian components in the wave beam propagated in the launcher.

In addition to those listed above, numerous additional requirements may be formulated as well, e.g. shock resistance, acceptable thermal expansion values, etc. A relatively easy assessment of the impact that the listed requirements exert on the geometrical parameters of the launcher is possible by transforming Eqs. (6)-(13) in such a way that the field mode parameters $\left(n, m, \chi_{m n}{ }^{\prime}\right)$ appear in the equations and the Brillouin angle $\psi$ is an independent variable. After applying relatively simple transformations, design Eqs. (6)-(13) take the following form:

$$
\begin{gathered}
d=\frac{D}{\lambda_{0}}=\frac{\frac{\chi_{m n}{ }^{\prime}}{\sin \psi}=\frac{\chi_{m n}{ }^{\prime}}{\pi \sin \psi},}{\cos \theta=\frac{m}{\chi_{m n}}}, \\
\sin \theta=\sqrt{1-\left(\frac{m}{\chi_{m n}}\right)^{2}}, \\
R_{c}=a \cdot \cos \theta=a \frac{m}{\chi_{m n}} \\
L_{b}=2 a \cdot \sin \theta \cot \psi=2 a \sqrt{1-\left(\frac{m}{\chi_{m n}}\right)^{2}} \cot \psi, \\
\tau=\arctan \frac{\tan \psi}{\frac{\sin \theta}{\theta}}, \\
L=2 \pi a \cdot \cot \tau=2 \pi a \cdot \frac{\sin \theta}{\theta} \cot \psi .
\end{gathered}
$$

From Eqs. (18)-(20), one can eliminate waveguide radius $a$ by entering normalized quantities of caustic radius $r_{c}$, axial shift $L_{b}$, and length of the launcher $L$ :

$$
\begin{gathered}
r_{c}=\frac{R_{c}}{a}=\frac{m}{\chi_{m n}} \\
l_{b}=\frac{L_{b}}{D}=\sqrt{1-\left(\frac{m}{\chi_{m n}}\right)^{2}} \cot \psi, \\
l=\frac{L}{\pi D}=\frac{\sin \theta}{\theta} \cot \psi=\frac{\sqrt{1-\left(\frac{m}{\chi_{m n}}\right)^{2}}}{\arccos \frac{m}{\chi_{m n}}} \cot \psi .
\end{gathered}
$$

In Eq. (23), length $L$ of the launcher was normalized to circumference $\pi D$ of the waveguide constituting the input section of the launcher. The analytical form of Eq. (23) can be $\operatorname{simplified~by~entering~the~} \operatorname{sinc} \theta$ function:

$$
\operatorname{sinc} \theta=\frac{\sin \theta}{\theta} \text {. }
$$

The $\operatorname{sinc} \theta$ function, for relatively small values of the angle $\theta$ characterizing the high-order $T E_{m n}$ fields, takes values slightly lower than one, as shown in Eq. (23), so it affects the launcher length to a relatively small extent. Therefore, it may be treated as a correction factor for the launcher length normalized to the $\pi D$ circumference. The correcting factor of the launcher is expressed as:

$$
l_{k}=\frac{l}{\operatorname{sinc} \theta}=\cot \psi \text {. }
$$

Equations (14), (21), (22), and (25) allow for tracking the impact that the Brillouin angle $\psi$ and field type $\left(m, \chi_{m n}{ }^{\prime}\right)$ exert on normalized geometrical parameters of the launcher, such as waveguide diameter $d$, caustic radius $r_{c}$, axial shift $l_{b}$, and launcher length $l_{k}$.

In Appendices $\mathrm{A}$ and $\mathrm{B}$, direct dependencies between the launcher length and the waveguide circumference are derived.

\section{Launcher Design Supporting Graphs}

Based on the analytical formulas provided in Section 2, specific the graphs and curves have been drawn to facilitate the design of the launcher.

\subsection{Diagrams Presenting Dependence of Waveguide Diameter and Launcher Length on the Brillouin Angle}

In this paper, graphs of the functions showing the relationship between $d(\psi)$ and $l_{k}(\psi)$ have been prepared. These graphs, just like analytical formulas, allow to trace the impact of the Brillouin angle $(\psi)$ and field type $\left(m, \chi_{m n}{ }^{\prime}\right)$ on the launcher's geometrical parameters $d$ and $l_{k}$. This graphical presentation is, however, much more convenient to follow than analysis of Eqs. (14) and (25). The manner in which diagrams $d(\psi)$ and $l_{k}(\psi)$ are used in the launcher design process is presented below. Figure 7 shows 
the parametric graph of function $d(\psi)$ for many realistically selected parameter values $p=\frac{\chi_{m n}{ }^{\prime}}{\pi}$. This graph clearly visualizes the impact of the field mode and of the Brillouin angle value on the normalized waveguide diameter $d$.

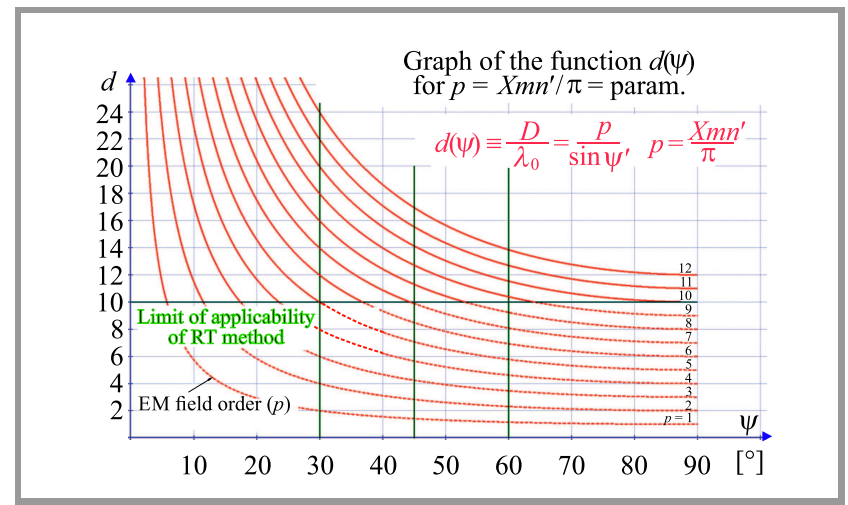

Fig. 7. Relationship between normalized waveguide diameter $d$ and the Brillouin angle $\psi$.

The graph contains the following information:

- analytical formula describing function $d(\psi), d(\psi) \equiv$ $\frac{D}{\lambda_{0}}=\frac{p}{\sin \psi}$, where $p=\frac{\chi_{m n}{ }^{\prime}}{\pi}=$ parameter,

- arbitrary limit of applicability of the RT method to the description of ray propagation in the launcher $\left(d_{\min }=10\right)$. For, $d>10$, errors in the analytical description of wave propagation taking place in the launcher have a satisfactorily low level of $1 \%$. The error rate decreases with an increase in $d$ [11],

- limits of the middle range of the Brillouin angle variation $\Delta(\psi)$ set arbitrarily at: $\psi_{\min }=30^{\circ}$ and $\psi_{\max }=60^{\circ}$. The Brillouin angle values adopted in projects are usually within that range,

- straight line marking the middle of the range $\Delta(\psi)$ : $\psi_{c}=45^{\circ}$,

- values of parameter $p$ for all curves of function $d(\psi)$,

- trend indicator determining the direction of the shift in characteristic $d(\psi)$ with the increase of in parameter $p$ (with the increase of the field mode order),

- parts of the $d(\psi)$ characteristics below the marked limit of applicability of the RT method (dashed line) cannot be used in this range because errors in the analytical description of wave propagation in the launcher are too large.

Variable $\psi$ is independent and its range covers all potential values of angle $\psi \in\left[0,90^{\circ}\right]$, while the range of the dependent variable $d$ is arbitrarily set to $d \in[0,26]$. The range of variation of parameter $p(p \in[1,12])$ was adopted arbitrarily, based on a review of the value of the Bessel function derivative zeroes $\chi_{m n}{ }^{\prime}$ for various field modes [16].

The $l_{k}(\psi)$ function graph, shown in Fig. 8, presents the impact of the $\psi$ angle value on the launcher length $l_{k}$.
Figure 8 contains the following information:

- analytical formula describing the $l_{k}(\psi)$ function $l_{k}(\psi) \equiv \frac{L}{\pi D \operatorname{sinc} \theta}=\cot \psi$,

- limits of the middle range of the Brillouin angle variation $\Delta(\psi)$, set arbitrarily at: $\psi_{\min }=30^{\circ}$ and $\psi_{\max }=60^{\circ}$,

- straight line marking the middle of the range $\Delta(\psi): \psi_{c}=45^{\circ}$

- ranges of Brillouin angle $\psi$ in which launcher shortening or elongation occurs in relation to its length at $\psi=45^{\circ}$.

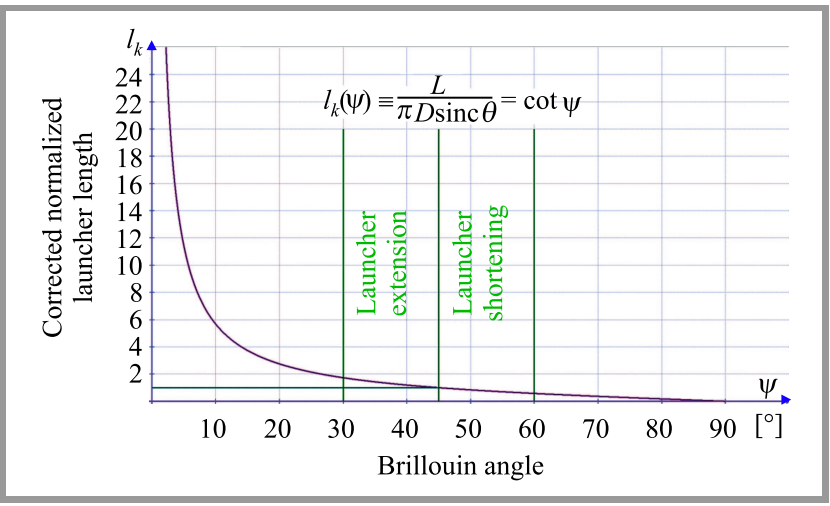

Fig. 8. Relationship between normalized launcher length $l_{k}$ and the Brillouin angle $\psi$.

Similarly to Fig. 7, independent variable $\psi$ covers all potential values of angle $\psi \in\left[0,90^{\circ}\right]$, while the range of the dependent variable $l_{k}$ is arbitrarily set to $l_{k} \in[0,26]$. There is a relatively small difference between $d$ and $l_{k}$ values for a given angle $\psi$. This makes it possible to apply the same ordinate axis for both of these quantities when plotting functions $d(\psi)$ and $l_{k}(\psi)$ in one common coordinate system (Fig. 9). Additionally, this method of presentation facilitates observation of the impact that field mode and the Brillouin angle $\psi$ have on the launcher's geometrical parameters $d$ and $l_{k}$.

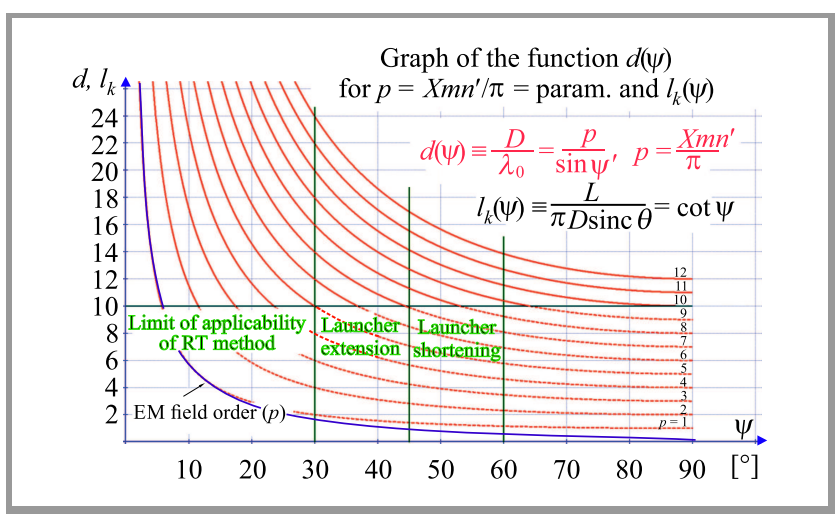

Fig. 9. Relationship between normalized launcher length $l_{k}$, normalized and corrected waveguide diameter $d$, and the value of Brillouin angle $\psi$. 


\subsection{Relationship Between sinc $(\theta)$ Function and Field Mode Parameters}

Equation (24) was transformed into Eq. (26) using Eqs. (15) and (16), and was then used to create the chart shown in Fig. 10.

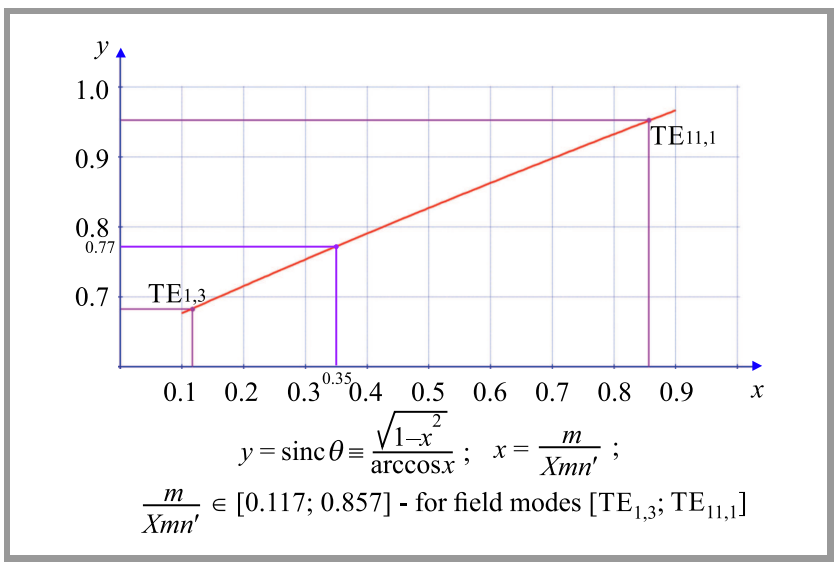

Fig. 10. Relationship between function $y=\operatorname{sinc} \theta$ and the field mode, represented by $x=\frac{m}{\chi_{m n}}$.

$$
y=\operatorname{sinc} \theta=\frac{\sin \theta}{\theta}=\frac{\sqrt{1-\left(\frac{m}{\chi_{m n}}\right)^{2}}}{\arccos \frac{m}{\chi_{m n}}} .
$$

The graph shown in Fig. 10 is a supplement to the graphs shown in Fig. 9. It shows the values of function $\operatorname{sinc} \theta$, referred to as field parameters, allowing the designer to quickly evaluate the function value for the adopted field mode. Knowledge of the function value allows to correct launcher length $l_{k}$, previously determined graphically in Fig. 9. The length corrected in accordance with Eq. (25) is equal to $l=l_{k} \cdot \operatorname{sinc} \theta$.

The range of variable $x=\frac{m}{\chi_{m n}}$ variation was determined after calculating its values for parameters $m$ and $\chi_{m n}{ }^{\prime}$ given in the paper [16]. The results of these calculations are presented in Table 1.

The review of values $m / \chi_{m n}{ }^{\prime}$ from Table 1 was relied upon to adopt the range of variable $x \in[0.1,0.9]$. The range of the dependent variable $y$ was adopted as $y \in[0.6,1]$, based on calculations of values for a given $x$. The two extreme points have been marked on the $\operatorname{sinc} \theta$ graph. They were described by field mode symbols corresponding to these values.

\section{Graph-assisted Launcher Design Method}

\subsection{Design Strategies}

As mentioned earlier, the operating frequency, the $T E$ field mode, and the geometrical parameters of a gyrotron cavity are usually basic input data for the launcher design process. With waveguide radius $a$ known, and with the knowledge of $\lambda_{0}$ and $m, n$, indexes of $T E_{m n}$ mode are sufficient to calculate all other parameters. Unfortunately, as one can see in Fig. 1, radius $a$ differs from the gyrotron cavity radius $a_{c}$, $\left(a>a_{c}\right)$. This requires that the cavity and the launcher be connected by a tapering transition section known as a "taper". As a result, there is some freedom in the selection of the waveguide radius in the launcher design. This allows for the creation of various design strategies that depend on additional requirements applicable to the launcher. One of the additional requirements consists in determining the proper ratio between the waveguide diameter and the wavelength, ensuring sufficiently high mode purity of the output Gaussian beam. The next requirement may consist, for instance, in determining the right length of the launcher selected, so that it does not appear on the trajectory of electrons moving to the collector (the so-called spent electrons).

\subsection{Launcher Design for Given Field Mode Indexes}

Initial input data:

- field mode: $T E_{95}$,

- frequency, (wavelength): $f_{0},\left(\lambda_{0}\right)$.

Design steps:

Step 1. Selection of the $d(\psi)$ characteristics with parameter $p$ closest to the setpoint.

For the assumed field mode $T E_{95}$ as $\chi_{m n}{ }^{\prime}=\chi_{95}{ }^{\prime}=25.8913$, parameter $p$ is calculated as $p=\frac{\chi_{m n^{\prime}}}{\pi}=8.24145676$. Within the range of characteristics $d(\psi)$ (waveguide diameter), we look for characteristics in which parameter $p$ is closest to the calculated value of 8.24145676 . This characteristic is

Table 1

Values of variable $x=\frac{m}{\chi_{m n}}$ for selected field modes $T E_{m n}$

\begin{tabular}{|r|c|c|c|c|c|c|c|c|c|c|c|c|}
\hline & $m=0$ & $m=1$ & $m=2$ & $m=3$ & $m=4$ & $m=5$ & $m=6$ & $m=7$ & $m=8$ & $m=9$ & $m=10$ & $m=11$ \\
\hline \hline$n=1$ & 3.832 & 1.841 & 3.054 & 4.201 & 5.317 & 6.415 & 7.501 & 8.578 & 9.647 & 10.71 & 11.77 & 12.83 \\
\hline$m / \chi_{m n}{ }^{\prime}$ & 0 & 0.543 & 0.655 & 0.714 & 0.752 & 0.779 & 0.800 & 0.816 & 0.829 & 0.840 & 0.850 & 0.857 \\
\hline$n=2$ & 7.016 & 5.330 & 6.706 & 8.015 & 9.282 & 10.52 & 11.73 & 12.93 & 14.11 & 15.29 & 16.45 & 17.60 \\
\hline$m / \chi_{m n}{ }^{\prime}$ & 0 & 0.188 & 0.298 & 0.374 & 0.431 & 0.475 & 0.511 & 0.541 & 0.567 & 0.588 & 0.608 & 0.625 \\
\hline$n=3$ & 10.17 & 8.536 & 9.969 & 11.35 & 12.68 & 13.99 & 15.27 & 16.53 & 17.77 & 19.00 & 20.22 & 21.43 \\
\hline$m / \chi_{m n}{ }^{\prime}$ & 0 & 0.117 & 0.200 & 0.264 & 0.315 & 0.357 & 0.393 & 0.423 & 0.450 & 0.474 & 0.494 & 0.513 \\
\hline
\end{tabular}


marked in the graph (Fig. 11) in magenta, with $p=8$. At this design stage, it is possible to move the operating point $P$ freely along the selected characteristic within the permitted range above the limit of applicability of the RT method. One may see that when point $P$ is moved upwards, waveguide diameter $\left(d \equiv D / \lambda_{0}\right)$ increases.

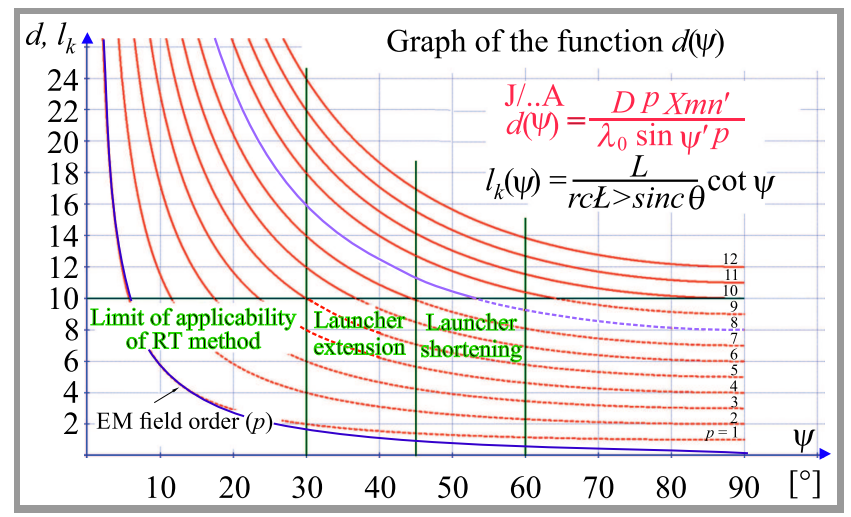

Fig. 11. Highlighted area shows the $d(\psi)$ characteristic with the $p$ parameter closest to the calculated value.

Step 2. Determining the operating point on the selected $d(\psi)$ curve.

As mentioned previously, the additional requirement consists in the need to determine the proper ratio between waveguide diameter and wavelength $D / \lambda_{0}$ for high Gaussian mode purity of the output beam. In this example, the $D / \lambda_{0}$ value is determined by the selection of the Brillouin angle value $\psi=45^{\circ}$, as shown in Fig. 12. The value read is $D / \lambda_{0}=11.33$. If this value is greater than the $D / \lambda_{0}$ of the gyrotron cavity, it will be necessary to apply the taper transition between the cavity and the launcher.

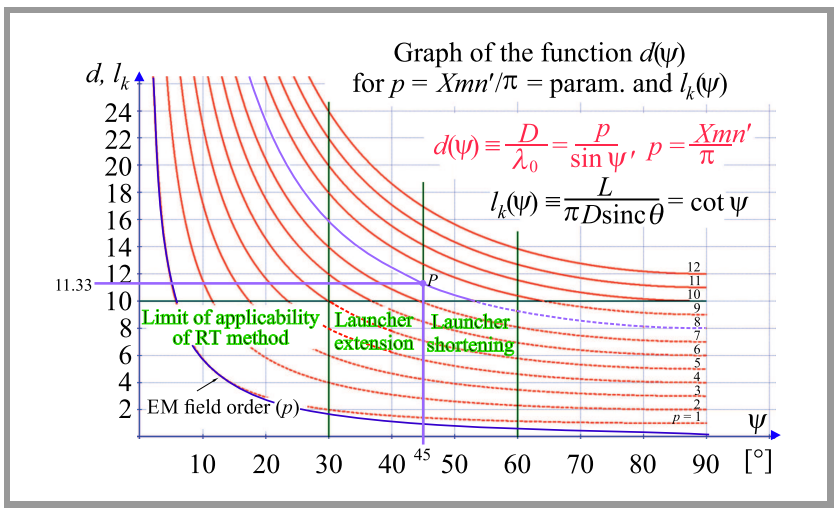

Fig. 12. Illustration of operating point $P$ selection based on characteristic $d(\psi)$.

Step 3. Determining the operating point on $l_{k}(\psi)$ curve. This curve illustrates the relationship between normalized launcher length $l_{k}$ and the Brillouin angle $\psi$. The operating point, designated as $P 1$, is positioned at the intersection between the vertical line $\psi=45^{\circ}$ and the characteristic $l_{k}(\psi)$, as shown in Fig. 13.

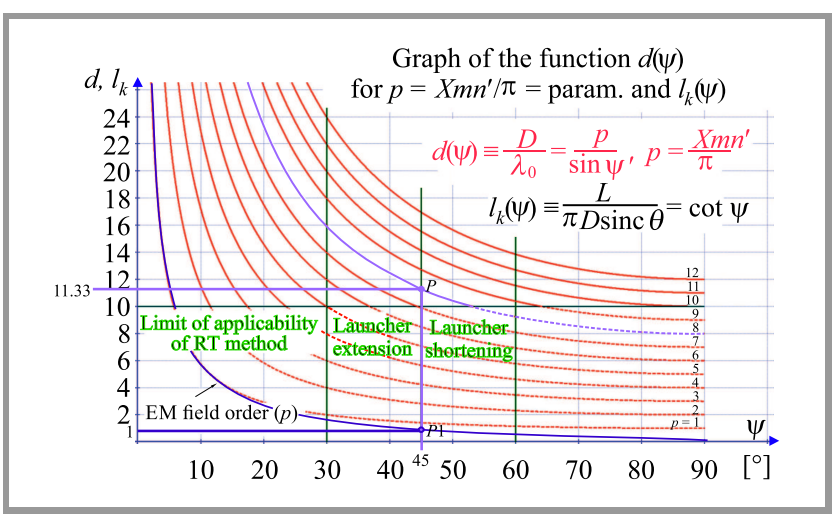

Fig. 13. Relationship between normalized launcher length $l_{k}$, normalized and corrected waveguide diameter $d$, and the value of Brillouin angle $\psi$.

Based on Eq. (25), the normalized value of corrected launcher length $l_{k}(\psi)$ is equal to 1 (the ordinate of point $P 1$ for $\psi=45^{\circ}$ ). Therefore, in order to determine normalized length $l$ of the launcher equal to Eq. (23), the ratio $L /(\pi D)$ and the value of the $\operatorname{sinc} \theta$ function should be calculated for the assumed field mode.

Step 4. Calculation and graphic visualization of the $\operatorname{sinc} \theta$ function value.

According to Eq. (8) and field parameters given in step 1:

$$
\theta=\arccos \frac{m}{\chi_{m n^{\prime}}}=\arccos \frac{9}{25.8913}=1.21[\mathrm{rad}],
$$

therefore: $\operatorname{sinc} \theta=0.771$.

To show the value of the sinc $\theta$ function, the plot of the function's value depending on the field mode, as presented in Fig. 10, will be used. In this plot, the value of the function calculated above is the ordinate of point $P$, as shown in Fig. 14.

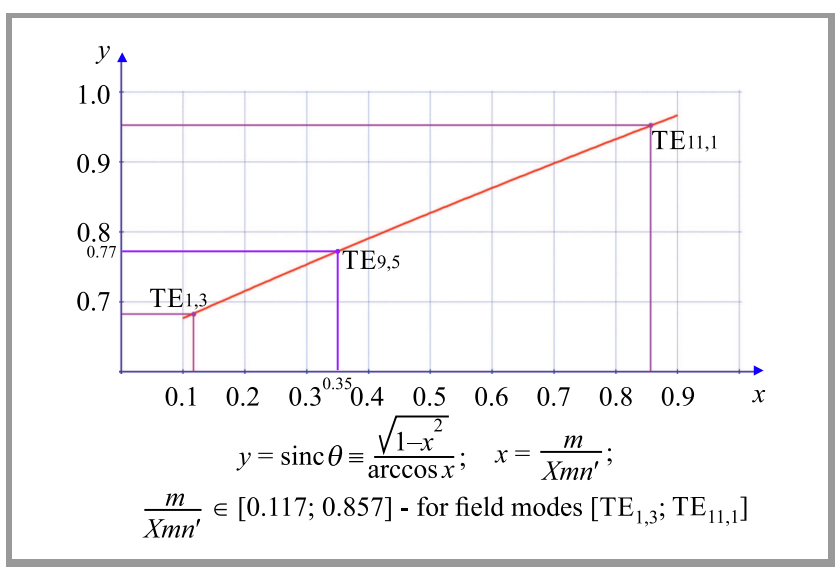

Fig. 14. Visualization of the value of the $y=\operatorname{sinc} \theta$ function for the selected field mode, represented by $x=\frac{m}{\chi_{m n}}$.

Step 5. Calculation of the ratio between launcher length $L$ and waveguide circumference $\pi D$.

This ratio is the standard length of the launcher $l=\frac{L}{\pi D}$, as introduced in Eq. (23). Based on Eq. (25) $l_{k}=\frac{l}{\operatorname{sinc} \theta}$, 
hence, $l=l_{k} \operatorname{sinc} \theta$. The normalized, corrected launcher length $l_{k}$ was determined in step 3 and is equal to 1 , while $\operatorname{sinc} \theta$, determined in step 4 , is 0.771 . So, $l=l_{k} \operatorname{sinc} \theta=$ 0.771 .

Step 6. Calculation of waveguide diameter $D$ for the assumed wavelength $\lambda_{0}$.

The value of $\frac{D}{\lambda_{0}}$ was determined in step 2 as the ordinate of point $P$. Hence, $D=y_{p} \lambda_{0}=11.33 \lambda_{0}$. For example:

- for $\lambda_{0}=3 \mathrm{~cm}\left(f_{0}=10 \mathrm{GHz}\right)$ : $D=11.33 \cdot 3=33.99 \mathrm{~cm}$,

- for $\lambda_{0}=1.875 \mathrm{~cm}\left(f_{0}=16 \mathrm{GHz}\right)$ : $D=11.33 \cdot 1.875=21.24 \mathrm{~cm}$.

Step 7. Calculation of launcher length $L$ for the assumed wavelength $\lambda_{0}$.

The normalized launcher length $l=\frac{L}{\pi D}$ has been calculated in step 5. Hence, $L=\frac{\pi}{D}$, where, based on step 6: $D=y_{P} \cdot \lambda_{0}$. So $L=\pi l D=\pi l y_{P} \cdot \lambda_{0}$. After substitution into this equation where according to step $5, l=0.771$, and step 2 and $6, y_{P}=11.33, L=\pi \cdot 0.771 \cdot 11.33 \cdot \lambda_{0}=27.45 \cdot \lambda_{0}$ is obtained. For example:

- for $\lambda_{0}=1.875 \mathrm{~cm}, f_{0}=16 \mathrm{GHz}$ : $\mathrm{L}=27.45 \cdot 1.875=51.47 \mathrm{~cm}$.

The results obtained in steps 6-7 conclude the main part of the design procedure and make it possible, as shown above, to conveniently and simply calculate the main parameters of the launcher: its length and waveguide diameter for a given operating frequency. These results can, therefore, be used to design launcher models operating at other frequencies and in other field modes.

Other launcher parameters, such as: $R_{c}, L_{B}, \tau$, and $v_{\text {exp }}$, can be calculated using analytical formulas (9)-(13).

\subsection{Launcher Design Method for Assumed Field TE Mode and Standard Circular Waveguide Diameter}

The task presented here can be treated as an extension of the previous task and aims to use a standard circular waveguide in the launcher in order to reduce the costs of implementing the launcher model operating at a different (usually lower) frequency. The following initial input data is used:

- field mode: $T E_{95}$,

- frequency: $f_{0} \cong 16 \mathrm{GHz}$,

- waveguide diameter: $D \cong 21.15 \mathrm{~cm}$, (determined in step 6 of the previous example),

- normalized waveguide diameter: $d_{p}=\frac{D}{\lambda_{0}}=11.33 \mathrm{~cm}$, (determined in step 2).

Design steps:

Step 1. Selection of the standard circular waveguide.

From the table of standard circular waveguide sizes, a C10 waveguide with a diameter of $D=21.514 \mathrm{~cm}$ and a nominal working band of $0.039-1.29 \mathrm{GHz}$ has been selected [17].
Its diameter is as close as possible to value $D$ assumed in the initial input data.

Step 2. Calculation of wavelength $\lambda_{0}$ and operating frequency $f_{0}$.

The normalized waveguide diameter assumed in initial input data is equal to $\frac{D}{\lambda_{0}}=11.33$. Hence: $\lambda_{0}=\frac{D}{11.33}=$ $1.90 \mathrm{~cm}$ and $f_{0}=15.80 \mathrm{GHz}$.

Step 3. Calculation of launcher length.

Similarly to step 7 of the previous example, $L=27.45 \cdot \lambda_{0}=$ $52.13 \mathrm{~cm}$.

Step 4. Calculation of caustic radius $R_{c}$.

The calculation will be performed for a standard waveguide with a diameter of $D=21.514 \mathrm{~cm}$ selected in step 1 and the value $\frac{m}{\chi_{m n}}=0.3476$ calculated in step 4 of the previous task.

The calculation is performed for a standard circular waveguide selected in step 1 with a diameter of $D=21.514 \mathrm{~cm}$ and by taking the values calculated in step 4: $\frac{m}{\chi_{m n}{ }^{\prime}}=0.3476$. $R_{c}=a \cdot \cos \theta=\frac{D}{2} \cdot \frac{m}{\chi_{m n}}=\frac{21.514}{2} \cdot 0.3476=3.74 \mathrm{~cm}$.

Step 5. Calculation of distance $L_{B}$ (traveled by the ray in the axial direction between two successive reflections from the waveguide wall).

The calculation will be performed for a standard circular waveguide selected in step 1 with the diameter of $D=21.514 \mathrm{~cm}$. We then take the values calculated in step $4, \frac{m}{\chi_{m n}}=0.3476$, for which $\theta=\arccos \frac{m}{\chi_{m n}}=$ $\arccos \frac{9}{25.8913}=1.21[\mathrm{rad}]$. Hence, $\sin \theta=\sin 1.21=0.94$, and from Eq. (19), $L_{b}=D \sin \theta \cot \psi=21.514 \cdot 0.94$. $\cot 45^{\circ}=20.17 \mathrm{~cm}$.

Step 6. Calculation of helix inclination angle $\tau$.

For $\psi=45^{\circ}$ and $\operatorname{sinc} \theta=0.771$ :

$\tau=\arctan \frac{\tan \psi}{\operatorname{sinc} \theta}=0.91[\mathrm{rad}]=52.35^{\circ}$.

As expected, the helix inclination angle $\tau$ is slightly larger than the Brillouin angle $\psi$.

\section{Conclusions}

This paper presents an effective method for designing a Vlasov launcher that is a part of the gyrotron's microwave power transmission system. The method uses a graphical representation of known analytical relationships describing RT power transmission in such a system. An image of the design space makes it much easier for an engineer to take optimal decisions in the design process. In particular, for a given EM field mode $\left(m, \chi_{m n}{ }^{\prime}\right)$, the engineer can efficiently, easily and quickly determine the optimal launcher duty points: point $P$ (Fig. 13), Brillouin angle $\psi$, diameter $d$ of the launcher, and point P1 specifying length $l_{k}$ of the launcher. They can also assess the impact of the operating points' displacement on the launcher's geometrical parameters and determine the allowed displacement ranges. The method was presented based on the example of a Vlasov launcher with a helical cut, but its assumptions may be used for all other launcher geometries. At the final stage of the design process, the described method allows to calculate the launcher geometry taking into account the wavelength. 
This is an additional advantage of the method concerned, resulting from the normalization of dimensions $d$ and $l_{k}$ depending on the wavelength.

The relatively large degree of design freedom offered by this method may greatly facilitate the design of the launcher, with additional requirements, such as those related to the limited installation space inside the gyrotron taken into consideration. Examples with detailed steps are presented, describing the design procedure relying on the method concerned. The data used in these examples, i.e. field mode $\left(\mathrm{TE}_{95}\right)$ and wavelength $\left(\lambda_{0}=3\right.$ and $\left.1.875 \mathrm{~cm}\right)$, were adopted arbitrarily. It was also shown how to use the presented graphs in order to easily implement a standard-size circular waveguide into the launcher design. The geometrical parameters of the launcher determined in the examples for the assumed values of $\lambda_{0}$ can be immediately recalculated for other values of $\lambda_{0}$, e.g. for typical terahertz wavelengths at which gyrotrons usually operate.

The correctness of the design procedures developed in this paper was verified by comparing the results of the Vlasov launcher design procedures described in previously published works with the results obtained, for the same input data, after implementing the design procedures this paper is concerned with. Such comparisons were made for projects presented in [7] and [15], containing also experimental verification of the radiators developed and confirming the usefulness of the design procedures devised.

\section{Appendix A}

\section{Direct relationship between launcher length and waveguide circumference}

Equations (27) and (28) describe the relationships between waveguide diameter and launcher length on the one hand, and the Brillouin angle on the other. It is possible to displace variable $\psi$ from the system of these equations. In this way, we obtain a direct relationship between these two quantities without mediating the Brillouin angle $\psi$.

$$
\begin{gathered}
d=\frac{D}{\lambda_{0}}=\frac{\frac{\chi_{m n}{ }^{\prime}}{\pi}}{\sin \psi}=\frac{\chi_{m n}{ }^{\prime}}{\pi \sin \psi}, \\
l=\frac{L}{\pi D}=\frac{\sin \theta}{\theta} \cot \psi=\operatorname{sinc} \theta \cot \psi,
\end{gathered}
$$

where:

$$
\frac{d^{2}}{p^{2}}-\frac{l^{2}}{(\operatorname{sinc} \theta)^{2}}=1
$$

or

$$
l=\operatorname{sinc} \theta \cdot \sqrt{\left(\frac{d}{p}\right)^{2}-1} .
$$

Equation (30), after renormalization of $d$ and $L$, takes the form of:

$$
L=\operatorname{sinc} \theta \cdot \sqrt{\left(\frac{\frac{D}{\lambda_{0}}}{p}\right)^{2}-1} \cdot \pi D .
$$

This relationship shows that for $L>0$, the condition $D / \lambda_{0}>p$ must be met. The smallest zero value of the derivative of the Bessel function is $\chi_{m n}{ }^{\prime}=1.8412$ [16]. Thus, it follows that the lowest value of parameter $p=\frac{\chi_{m n}{ }^{\prime}}{\pi}$ is equal to $p_{\min }=0.58607216$ and, consequently, that the smallest value of $D / \lambda_{0}$ should be greater than 0.58607216 . This is a milder condition than that which determines the use of the RT method (requiring that $D / \lambda_{0}>10$ ). This condition is always met when designing the launcher using the RT method.

\section{Appendix B}

\section{Direct relationship between launcher length and waveguide diameter for $\psi=45^{\circ}$}

From the Eq. (27) for $\psi=45^{\circ}$ we get:

$$
\frac{d_{45}}{p}=\frac{2}{\sqrt{2}}
$$

but from Eq. (30):

$$
l_{45}=\operatorname{sinc} \theta \cdot \sqrt{\left(\frac{d_{45}}{p}\right)^{2}-1}=\operatorname{sinc} \theta
$$

which can also be seen from the Eq. (28):

$$
l=\operatorname{sinc} \theta \cot \psi
$$

Because, $\cot 45^{\circ}=1$, so $l_{45}=\operatorname{sinc} \theta$. Therefore,

$$
d_{45}=\frac{D_{45}}{\lambda_{0}}=\frac{\sqrt{2}}{\pi} \cdot \chi_{m n}{ }^{\prime},
$$

and

$$
l_{45}=\frac{L_{45}}{\pi D_{45}}=\operatorname{sinc} \theta .
$$

Hence, after renormalization:

$$
D_{45}=\frac{\sqrt{2}}{\pi} \cdot \chi_{m n}{ }^{\prime} \cdot \lambda_{0}
$$

and

$$
L_{45}=\sqrt{2} \cdot \chi_{m n}{ }^{\prime} \cdot \operatorname{sinc} \theta \cdot \lambda_{0}
$$




\section{References}

[1] C.-H. Du, X.-B. Qi, and P.-K. Liu, "Theoretical study of a broadband quasi-optical mode converter for pulse gyrotron devices", IEEE Transac. on Plasma Sci., vol. 44, no. 10, pp. 2348-2355, 2016 [Online]. Available: http://ieeexplore.ieee.org/document/7572128/ (DOI: 10.1109/TPS.2016.2606497).

[2] Z. Li and J. Feng, "Design of a Vlasov mode converter of $263 \mathrm{GHz}$ gyrotron oscillator for DNP-NMR", The J. of Engineer., vol. 2018, no. 14 , pp. 709-713, 2018 [Online]. Available: https:// digital-library.theiet.org/content/journals/10.1049/joe.2018.0124

[3] M. K. Alaria, N. Singh, U. Singh, A. Bera, and A. K. Sinha, "Development of $170 \mathrm{GHz}, 0.1 \mathrm{MW}$ short pulse gyrotron", in Proc. Fusion Engineer. and Design, vol. 144, 2019, pp. 87-92 [Online]. Available: https://doi.org/10.1016/j.fusengdes.2019.04.073

[4] M. Pilossof and M. Einat, "High-average-power second harmonic w-band gyrotron with room-temperature solenoid", IEEE Transac. on Electron Devices, vol. 67, no. 4, pp. 1804-1807, 2020 (DOI: 10.1109/TED.2020.2971653).

[5] B. Hui-Qi et al., "Analysis of a broadband quasi-optical mode converter for gyrotrons working in multi modes", in Proc. IEEE Asia Pacific Microwave Conf. (APMC), Kuala Lumpur, Malaysia, 2017 (DOI: 10.1109/APMC.2017.8251547).

[6] C. Gao et al., "Frequency-chirped dynamic nuclear polarization with magic angle spinning using a frequency-agile gyrotron", J. of Magnetic Resonance, vol. 308, 2019

(DOI: 10.1016/j.jmr.2019.106586).

[7] M. K. Alaria, A. K. Sinha, and H. Khatun, "Design and development of mode launcher for high frequency gyrotron", Infrared Physics \& Technol., vol. 75, pp. 187-192, 2016 (DOI:10.1016/j.infrared.2015.12.011).

[8] C. Zhang, W. Fu, and Y. Yan, "Study on a gyrotron quasi-optical mode converter for terahertz imaging", J. of Electromagnetic Waves and Applications, vol. 35, no. 2, pp. 176-184, 2020 (DOI: 10.1080/09205071.2020.1828186).

[9] X. Li, "Study of High-harmonic Gyro-devices in the THz Range", $\mathrm{Ph}$.D. Thesis, School of Electronic Engineering and Computer Science, Queen Mary University of London, London, UK, 2016 [Online]. Available: https://qmro.qmul.ac.uk/xmlui/bitstream/ handle/123456789/23214/LI_Xiang_FinalPhD_171016.pdf

[10] T. Kariya et al., "Development of over-MW gyrotrons for fusion at $14 \mathrm{GHz}$ to sub-THz frequencies", Nuclear Fusion, vol. 57, no. 6, 2017 [Online]. Available: https://iopscience.iop.org/article/ 10.1088/1741-4326/aa6875

[11] J. Jin, "Quasi-Optical Mode Converter for a Coaxial Cavity Gyrotron”, Forschungszentrum Karlsruhe, Karlsruhe, no. 7264, 2007 [Online]. Available: https://publikationen.bibliothek.kit.edu/ 270067871/3814966 [in German].

[12] S. N. Vlasov and I. M. Orlova, "Quasioptical transformer which transforms the waves in a waveguide having a circular cross section into a highly directional wave beam", Radiophysics and Quantum Electronics, vol. 17, no. 1, pp. 115-119, 1974 [Online]. Available: http://link.springer.com/10.1007/BF01037072

[13] L. A. Vainshtein, Open Resonators and Open Waveguides. Boulder, Colorado: Golem Press, 1969.

[14] V. M. Babic and V. S. Buldyrev, Short-Wavelength Diffraction Theory. Moscow: Springer-Verlag Berlin Heidelberg, 1972 (ISBN: 9783642834615).

[15] M. Blank, "High Efficiency Quasi-Optical Mode Converters for Overmoded Gyrotrons", Ph.D. Thesis, Massachusetts Institute of Technology, Department of Electrical Engineering and Computer Science, Massachusetts, 1994 [Online]. Available: https://dspace.mit.edu/bitstream/handle/1721.1/34089/ 32053304-MIT.pdf

[16] C. A. Balanis, Advanced Engineering Electromagnetics, 2nd Edition. Wiley and Sons, 2012 (ISBN: 9780470589489).
[17] H. Nickel, "Cross reference for hollow metallic waveguides", Spinner, 2020 [Online]. Available: https://www.spinner-group.com/ images/download/technical_documents/SPINNER_TD00036.pdf

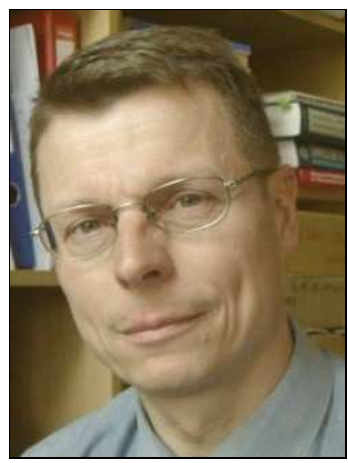

Grzegorz Jaworski received his Ph.D. degree from Wrocław University of Science and Technology in 1999. Between 2003 and 2004, he was with the Ørsted-DTU/EMI Department of Denmark Technical University, working on next generation SAR systems. Between 2006 and 2007, he participated in developing antennas for the Columbus module of the International Space Station. Currently, he is an Assistant Professor at the Electronics and Telecommunication Department, Faculty of Electronics, at Wrocław University of Science and Technology. Currently, his interests focus on high frequency techniques and technologies for telecommunications, radars, industry and medical applications.

(iD) https://orcid.org/0000-0002-9172-7437

E-mail: grzegorz.jaworski@pwr.edu.pl

Wrocław University of Science and Technology

Wybrzeże Wyspiańskiego 27

50-370 Wrocław, Poland

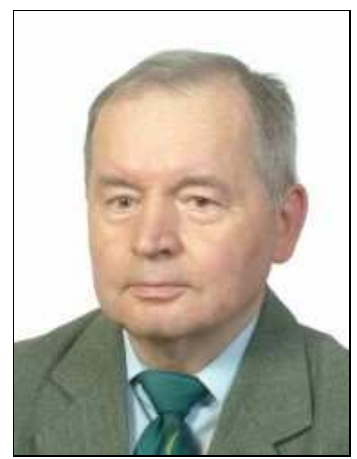

Andrzej R. Francik received his M.Sc. Eng. in Electronics from Wrocław University of Technology, Poland, in 1969. He started working as a university lecturer in 1970, and then as a research worker employed by the Institute of Telecommunications and Acoustics. His publications focus primarily on theoretical and practical problems related to the microwave module of ESR spectrometers. In 1978, Francik earned his Ph.D.E.E. for analyzing systematic distortions originating from the microwave unit. His research interests include power combiners, combgenerators, mixers, detectors, MICs, microwave instruments and, recently, gyrotron technologies. In 1992, he received a D.Sc. degree for publishing a monography titled "Instrumental Effects in Homodyne Electron Paramagnetic Resonance Spectrometers" (Ellis Horwood \& PWN, Chichester, Warszawa, 1989). In 2001, he was employed as an Associate Professor at his mother Institute. Currently, he is working, as a Professor Emeritus specializing in the terahertz technology.

(iD) https://orcid.org/0000-0001-6414-7106

E-mail: andrzej.francik@pwr.edu.pl

Wrocław University of Science and Technology

Wybrzeże Wyspiańskiego 27

50-370 Wrocław, Poland 


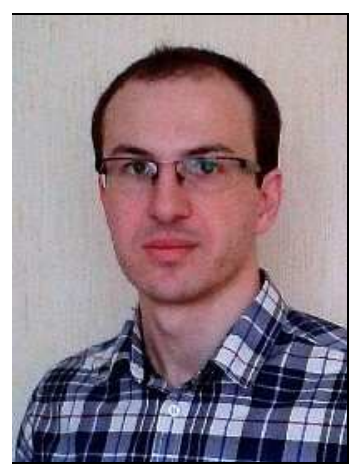

Maciej Nowak received his Ph.D. from Wrocław University of Science and Technology in 2016. He is currently an Assistant Professor at the Telecommunications and Teleinformatics Department, Faculty of Electronics, Wrocław University of Science and Technology. He is a member of the Wrocław Terahertz Center. His research interests include: terahertz spectroscopy, spectral imaging techniques and machine learning.

(iD) https://orcid.org/0000-0002-7747-4867

E-mail: maciej.nowak@pwr.edu.pl

Wrocław University of Science and Technology

Wybrzeże Wyspiańskiego 27

50-370 Wrocław, Poland

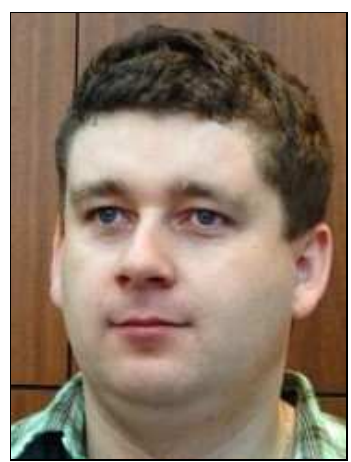

Kacper Nowak received his Ph.D. from Wrocław University of Science and Technology in 2012. He is currently an Assistant Professor at the Electronics and Telecommunication Department, Faculty of Electronics, Wrocław University of Science and Technology. His research interests include: terahertz spectroscopy, gyrotron technology, industrial automation, networking and programming.

(iD https://orcid.org/0000-0002-5980-8237

E-mail: kacper.nowak@pwr.edu.pl

Wrocław University of Science and Technology

Wybrzeże Wyspiańskiego 27

50-370 Wrocław, Poland 